\title{
A Mobile Detector for Muon Measurements Based on Two Different Techniques
}

\author{
B. Mitrica, ${ }^{1}$ D. Stanca, ${ }^{1}$ M. Petcu, ${ }^{1}$ I. M. Brancus, ${ }^{1}$ R. Margineanu, ${ }^{1}$ \\ A. Apostu, ${ }^{1}$ C. Gomoiu, ${ }^{1}$ A. Saftoiu, ${ }^{1}$ G. Toma, ${ }^{1}$ H. Rebel, ${ }^{2}$ A. Haungs, ${ }^{2}$ \\ O. Sima, ${ }^{3}$ A. Gherghel-Lascu, ${ }^{1}$ and M. Niculescu-Oglinzanu ${ }^{1}$ \\ ${ }^{1}$ Horia Hulubei National Institute for Physics and Nuclear Engineering, 077125 Magurele, Romania \\ ${ }^{2}$ Institute of Experimental Nuclear Physics, Karlsruhe Institute of Technology-Campus North, 76021 Karlsruhe, Germany \\ ${ }^{3}$ Department of Physics, University of Bucharest, 077125 Magurele, Romania
}

Correspondence should be addressed to B. Mitrica; mitrica@nipne.ro

Received 25 October 2012; Accepted 17 February 2013

Academic Editor: Maury Goodman

Copyright (c) 2013 B. Mitrica et al. This is an open access article distributed under the Creative Commons Attribution License, which permits unrestricted use, distribution, and reproduction in any medium, provided the original work is properly cited.

Precise measurements of the muon flux are important for different practical applications, both in environmental studies and for the estimation of the water equivalent depths of underground sites. A mobile detector for cosmic muon flux measurements has been set up at IFIN-HH, Romania. The device is used to measure the muon flux on different locations at the surface and underground. Its first configuration, not used in the present, has been composed of two $1 \mathrm{~m}^{2}$ scintillator plates, each viewed by wave length shifters and read out by two Photomultiplier Tubes (PMTs). A more recent configuration, consists of two $1 \mathrm{~m}^{2}$ detection layers, each one including four $1 \cdot 0,25 \mathrm{~m}^{2}$ large scintillator plates. The light output in each plate is collected by twelve optical fibers and then read out by one PMT. Comparative results were obtained with both configurations.

\section{Introduction}

The knowledge of cosmic ray muon flux is important in many applications, from low background measurements and the estimation of water equivalent depth for different underground locations to the characterization of the background in the vicinity of mega detectors for neutrino physics, like Super-Kamiokande or the future LAGUNA project [1], also for numerous environmental experiments like the solar activity characterization or the weather and climate change surveillance [2].

Also, it has successfully been used as muon tomography technique in the search for hidden rooms in pyramids [3] or in volcanology [4]. It has a long list of possible applications, like the detection of unknown caverns, to increase the safety procedures in mining excavations, in the oil industry as an easy way to search for oil bags or at the customs checkpoints, to scan the passing vehicles.

A mobile detector for measuring the muon flux is in use at IFIN-HH Bucharest [5]. Placed in a van for mobility, it interprets the coincidence events between two overlaid active layers as passing muons. A new configuration of the active layers, using optical fiber readout of the plastic scintillator sheet, was implemented and tested, and its performances were compared with the previous one, which used waveguides for the light collection. Using this new developed configuration, the possibility of directional measurements of muon flux was observed.

\section{The Apparatus}

The detection principle of the muon mobile detector is to identify the traversing muons as coincidence events between two overlaid $1 \mathrm{~m}^{2}$ active layers of scintillator material. Used for the current detector, two different concepts for the active layer are presented in Figure 1. One important property of this device is its mobility, the device being conceived to measure the muon flux at different locations at the surface of the ground or in underground. 


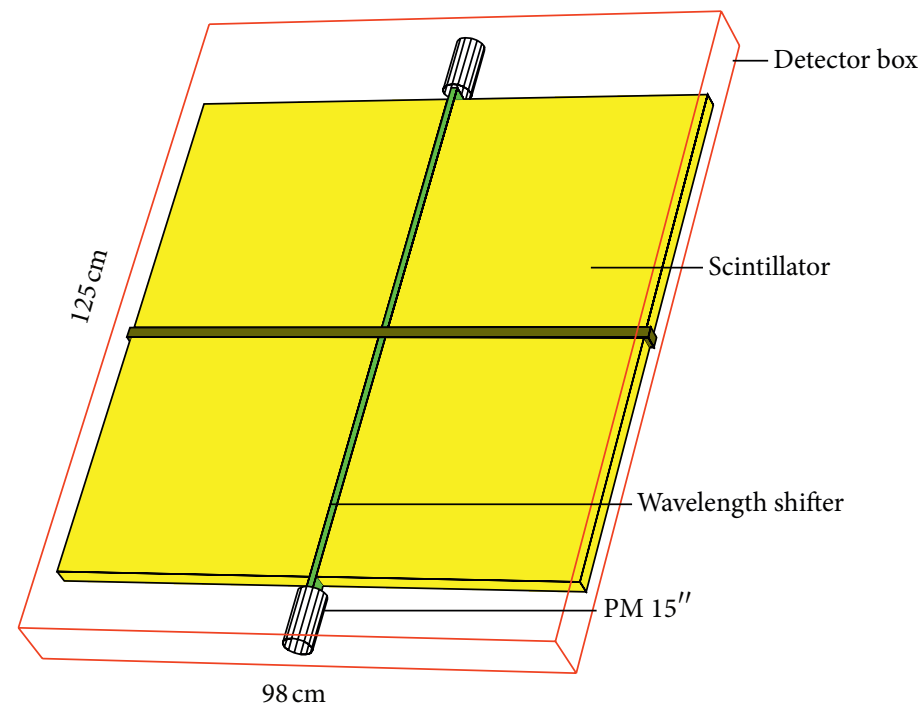

(a)

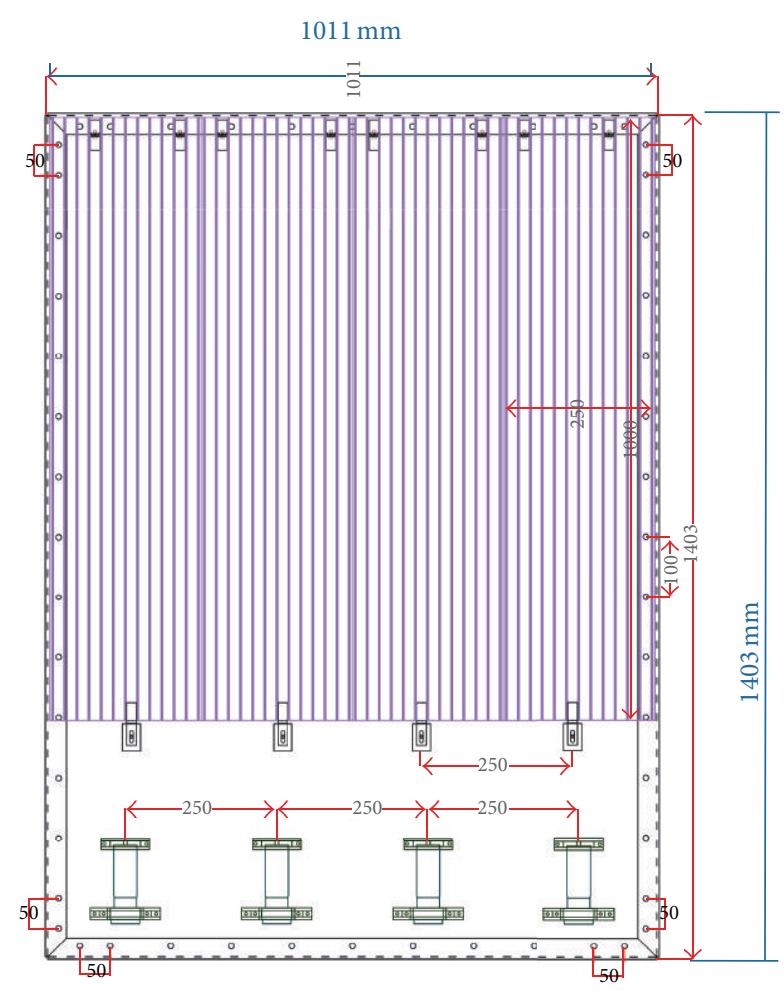

(b)

FIgURE 1: The detection module of (a) the first detector setup. Design of KASCADE [3]; (b) the new detector setup [5].

For the former configuration of the mobile detector, two detection modules were used, each module being a scintillator plate (NE114 type) of $0.9025 \mathrm{~m}^{2}$ and $3 \mathrm{~cm}$ thickness. Divided into four parts $\left(0.475 \cdot 0.475 \mathrm{~m}^{2}\right)[6,7]$, it is readout through a wavelength shifter (NE174A type) by two photomultiplier tubes (EMI 9902 type), as we can see in Figure 1(a).

The present configuration consists of two detection modules, each composed of 4 plastic scintillator sheets (Polystyrol $80 \%$, Methylmethacrylate $20 \%$ ) having $100 \cdot 25 \cdot 1 \mathrm{~cm}^{3}$. Every sheet is crossed by 13 longitudinal strips, 12 of them being filled with an optical fiber (Figure 1(b)). The light signal of each sheet is readout by a PMT. The modules are arranged on top of each other (at $30 \mathrm{~cm}$ distance). The signals from the eight photomultiplier tubes are OR-ed four by four $(1+2+$ $3+4)$ and $(5+6+7+8)$ and then measured in coincidence using a gate of $50 \mathrm{~ns}$, so no correction due to the dead time of the detector is necessary. A counter module registers the coincidence events (see Figure 2).

The calibration has been made by comparing the measured energy deposit spectrum of the minimum ionizing particles with GEANT simulated responses. The comparison of the measured and simulated spectra, of both configurations, is shown in Figure 3. The difference in the most probable energy deposit between the two configurations comes from the different thicknesses of the plates $(3 \mathrm{~cm}$ versus $1 \mathrm{~cm})$.

For the first configuration, having the most probable energy deposit at $6.3 \mathrm{MeV}$, the signal threshold was set to $2.1 \mathrm{MeV}$ [5]. For the new configuration, with the most probable energy deposit of $2.4 \mathrm{MeV}$, a signal threshold of $1.8 \mathrm{MeV}$ is set.

Considering the fact that not all muons that interact with the first layer manage to pass to the second one, due to scattering loses in between, the acceptance of the detector was also investigated, using GEANT4 code (see Figure 4). The muon flux at the surface of the ground was estimated using CORSIKA code [8], using the primary cosmic ray spectrum of proton and helium, obtained from AMS results during a space shuttle mission [9].

A correction factor $a^{*}=1.56$ has to be applied on the observed muon rate at ground level. The distance between the centers of the two active layers of the detector is $30 \mathrm{~cm}$. For a distance of $8 \mathrm{~cm}$, obtained if the two active layers are overlapping, a correction factor $a^{\prime}=1.11$ is obtained. No correction factor due to the geometry of the detector's surface is required. The corrected muon flux is given by

$$
\Phi_{\mu}=a^{*} \cdot R
$$

where $R$ is the counting rate of the detector and $\Phi_{\mu}$ is the real flux.

\section{Measurements and Results}

Using the mobility of the detector, measurements of cosmic muon flux have been performed for both configurations of the active layers at different locations on the surface with different elevations. The results, presented in Table 1, are 


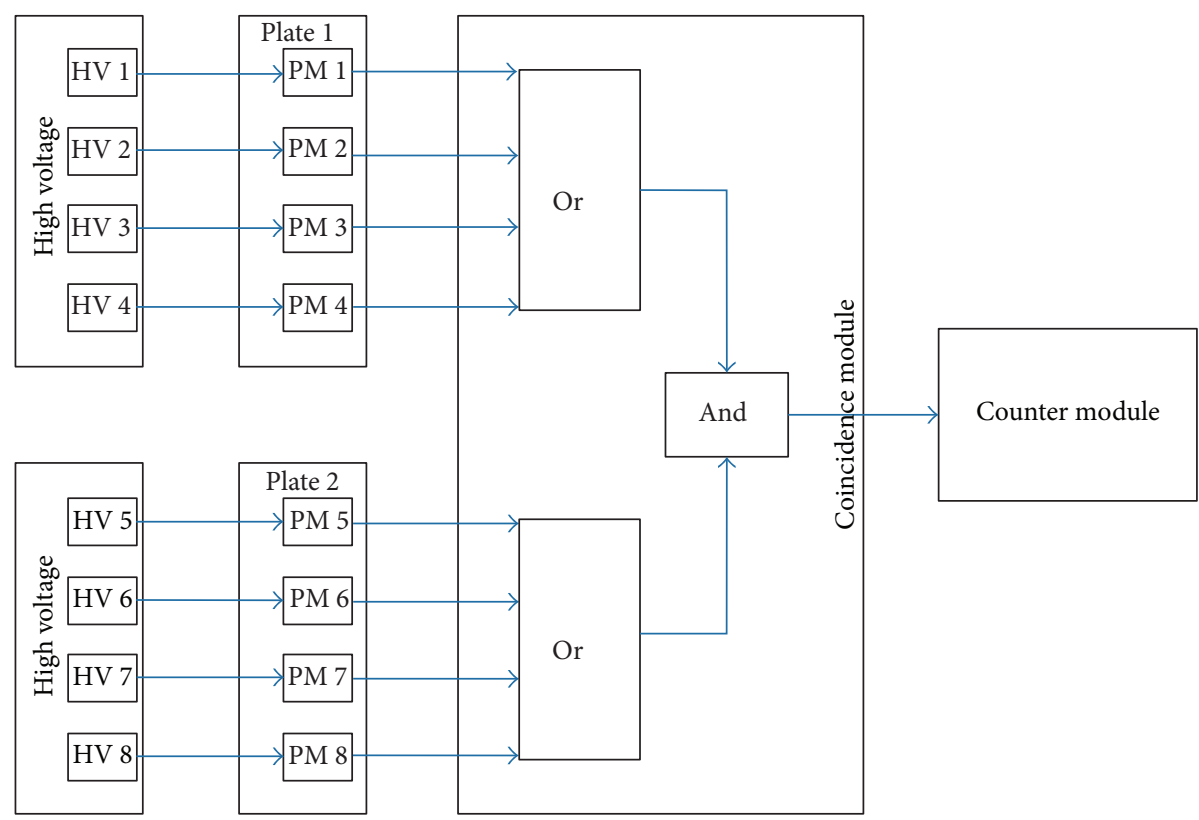

FIgURE 2: The schematic view of the electronic detection system.

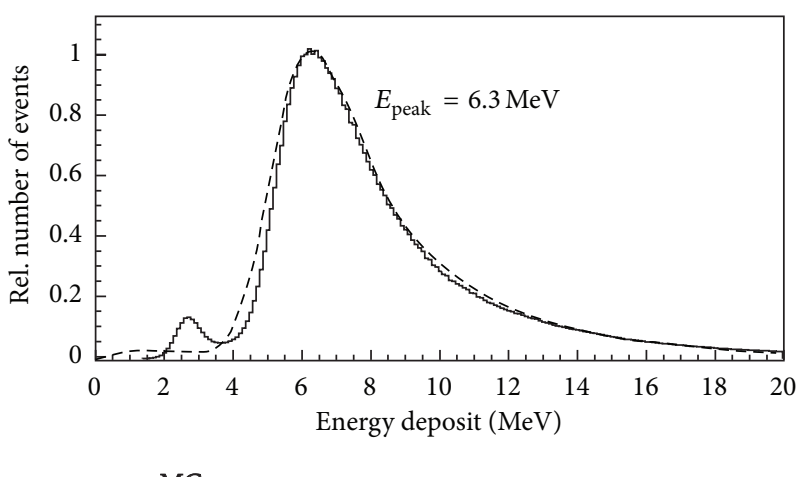

$---\mathrm{MC}$

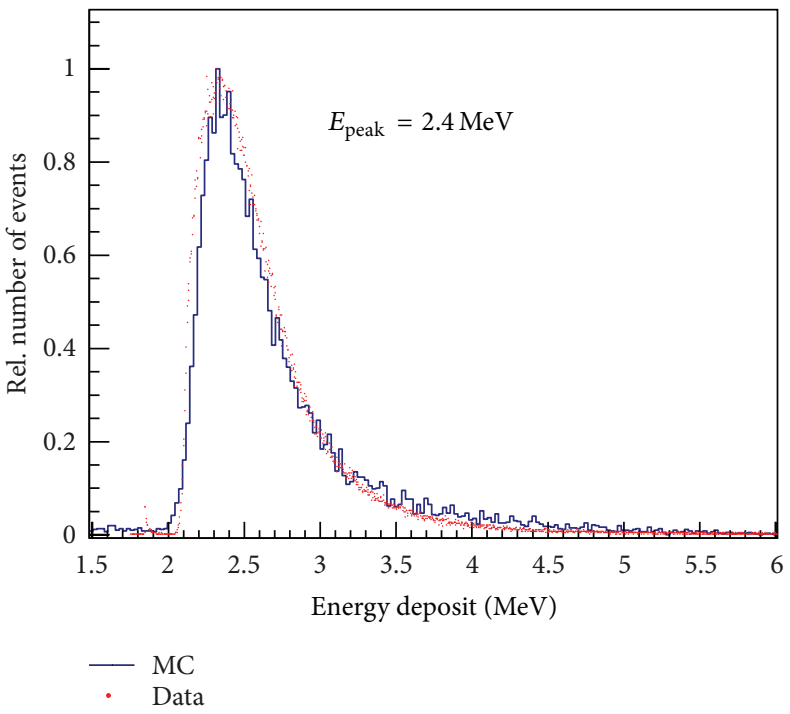

(b)

FIGURE 3: (a) The energy calibration for one scintillator plate of the previous configuration, made by comparing the measured energy deposit spectrum of the minimum ionizing particles with GEANT 3.21 [28] simulated one. (b) The energy calibration for one scintillator sheet of the recent detector setup, made by comparison of the measured energy deposit spectrum of the minimum ionizing particles with GEANT 4.94 [29] simulated one.

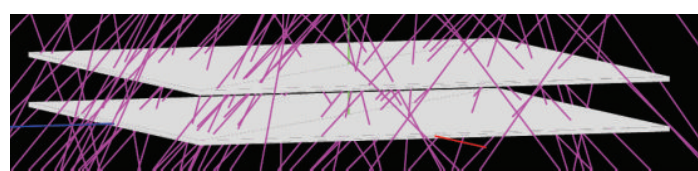

FIGURE 4: The schematically view of the muons interacting with the new detection system, observed with GEANT 4.94 code (magenta lines represent muons). We can see that not all muons that cross the first scintillator layer will pass through the second one. compared with those previously reported in [5] and displayed in Figure 5.

The acquisition time for each data set was $1 \mathrm{~h}$, all runs being performed at approximately the same time of the day (noon) in order to reduce the eventual influence of the solar activity and of atmospheric conditions.

Measurements of the cosmic muon flux have been performed also underground in the Unirea mine from Slanic 
TABLE 1: Cosmic muon flux measured at different geographic locations with different elevations, using both configurations of the active layers. The altitude and the coordinates were measured with a GPS system.

\begin{tabular}{lcccc}
\hline & $\begin{array}{c}\text { Latitude } \\
\left({ }^{\circ}\right)\end{array}$ & $\begin{array}{c}\text { Longitude } \\
\left({ }^{\circ}\right)\end{array}$ & $\begin{array}{c}\text { Altitude } \\
(\mathrm{m} \text { a.s.l. })\end{array}$ & $\begin{array}{c}\text { Muon flux } \\
\left(\mathrm{m}^{-2} \mathrm{~s}^{-1}\right)\end{array}$ \\
\hline \multirow{4}{*}{ Former } & 44.36 & 28.05 & $7 \pm 5$ & $119.1 \pm 3.6$ \\
configuration & 44.40 & 26.10 & $64 \pm 5$ & $122.3 \pm 3.7$ \\
& 44.32 & 28.19 & $70 \pm 5$ & $128.1 \pm 3.8$ \\
& 45.24 & 25.94 & $408 \pm 5$ & $143.2 \pm 4.3$ \\
& 45.28 & 25.97 & $588 \pm 5$ & $145.3 \pm 4.4$ \\
Recent & 45.29 & 25.94 & $655 \pm 5$ & $146.7 \pm 4.4$ \\
configuration & 44.97 & 26.02 & $219 \pm 5$ & $129.1 \pm 4.5$ \\
& 45.07 & 26.03 & $266 \pm 5$ & $136.7 \pm 4.7$ \\
& 45.24 & 25.94 & $438 \pm 5$ & $142.7 \pm 4.9$ \\
& 45.26 & 25.96 & $603 \pm 5$ & $143.4 \pm 4.9$ \\
& 45.48 & 25.97 & $693 \pm 5$ & $144.6 \pm 4.9$ \\
& 45.48 & 25.91 & $1145 \pm 5$ & $146.8 \pm 5.0$ \\
& 45.36 & 25.52 & $1338 \pm 5$ & $155.9 \pm 5.3$ \\
\hline
\end{tabular}

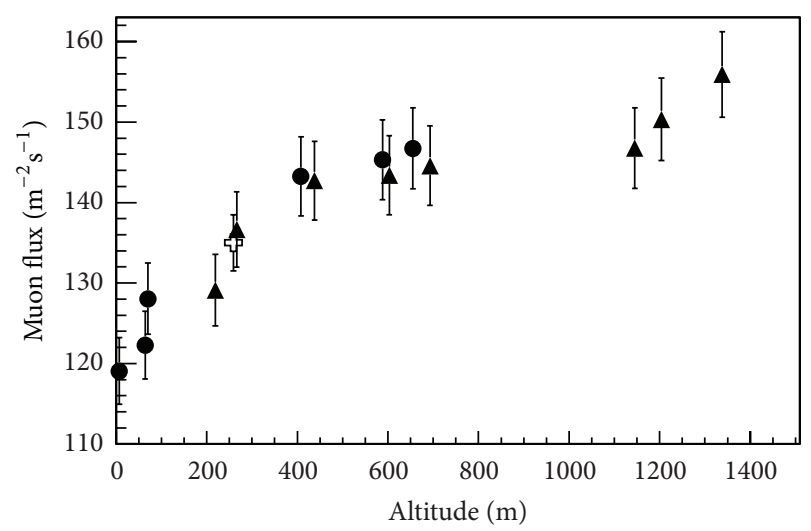

A New configuration data

- Former configuration data

↔ Results from reference [30]

FIGURE 5: Measured results of the muon flux variation with altitude, obtained with both detector configurations of the active layers of the detector. The circles represent the data obtained with the former configuration. The data obtained with the new configuration is presented with triangles. The cross point represents the results from [30].

Prahova (-208 $\mathrm{m}$ depth from surface), where a low-radiation level laboratory of IFIN-HH is set up, using both configurations. The detection modules were removed from the car and transported by an elevator to the observation level.

The responses are similar, $0.18 \pm 0.01 \mathrm{~m}^{-2} \mathrm{~s}^{-1}$ for the first configuration and $0.19 \pm 0.002 \mathrm{~m}^{-2} \mathrm{~s}^{-1}$ for the second one. The acquisition time was 1 hour for the first set of measurements and 10 hours for the second one.

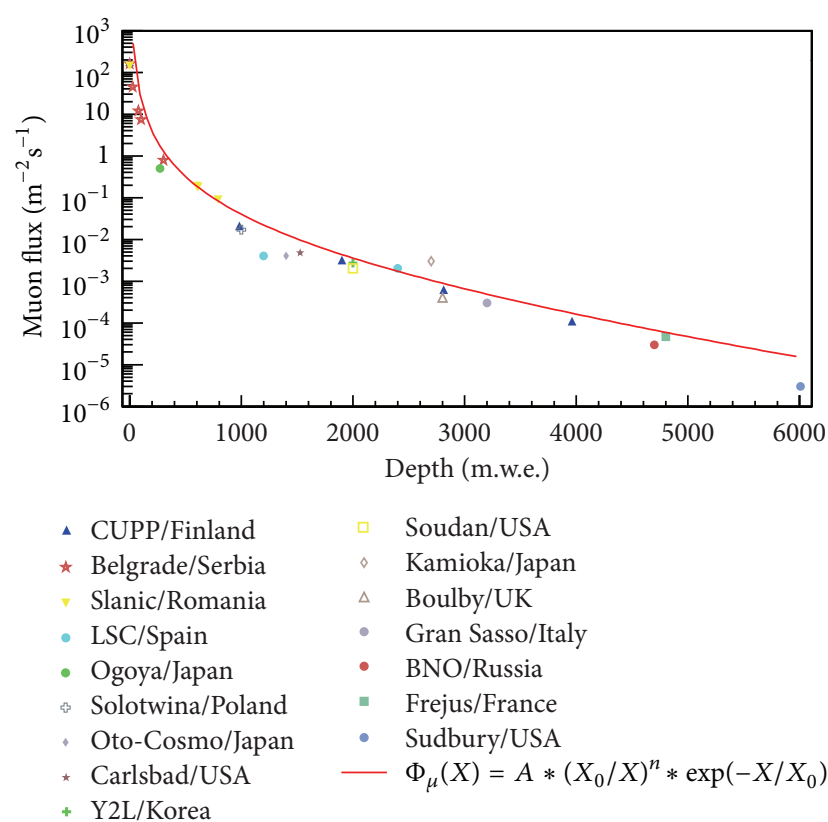

FIGURE 6: The variation of flux as function of mwe depth for the results presented in Table 2 .

The estimation of the water equivalent depth from muon flux data is made by the formula

$$
\Phi_{\mu}(X)=A *\left(\frac{X_{0}}{X}\right)^{n} * \exp \left(\frac{-X}{X_{0}}\right),
$$

with $A=0.03 \mathrm{~m}^{-2} \mathrm{~s}^{-1}$ being an overall normalization constant, $X_{0}=1470$ mwe representing an effective attenuation length for high-energy muons, and $n=2.5$ being a free parameter [9]. The variation of the muon flux with the water equivalent depth is still an open problem and one of our detector's goals. The formula has been obtained by Monte Carlo simulation using the MUSIC code [10].

A value of $610 \pm 11$ mwe was obtained using the flux measured with the first configuration; from the second one, a $597 \pm 10$ mwe is being calculated. We can see the evolution of the muon flux as a function of mwe depth (see Figure 6). The data are compared with others over the world (see Table 2).

In order to check if there are any preferential arrival directions of the muons, coincidence measurements have been performed in the underground between the scintillator sheets of the detector setup. The measurements have been performed in Unirea mine from Slanic Prahova salt ore, close to one of the mine's wall (see Figure 7). The "Unirea" mine is characterised by temperature: $12.0-13.0^{\circ} \mathrm{C}$, humidity: $65-$ $70 \%$, excavated volume: $2.9 \cdot 10^{6} \mathrm{~m}^{3}$, floor area: $7 \cdot 10^{4} \mathrm{~m}^{2}$, average high: $52-57 \mathrm{~m}$, aerosols $<10 \mathrm{~mm}: 2108 \mathrm{part} / \mathrm{m}^{3}$, distance between walls: $32-36 \mathrm{~m}$, and existing infrastructures: electricity, roads, railway, elevator, phone, Internet, and GSM networks (also inside the galleries). More details regarding the radioactive background in the mine are presented in [11].

The order of the scintillator sheets of the detector is shown in Figure 8. The results are presented in Table 3. The system was positioned in the cavern, so the sheets 1 and 5 are to be 
TABLE 2: A compilation of different muon flux measurements over the world.

\begin{tabular}{|c|c|c|c|c|}
\hline Location (laboratory)/country & Latitude $\left({ }^{\circ}\right)$ & Longitude $\left({ }^{\circ}\right)$ & Depth (mwe) & Muon flux $\left(\mathrm{m}^{-2} \mathrm{~s}^{-1}\right)$ \\
\hline CUPP/Finland [13] & $63.39 \mathrm{~N}$ & $26.02 \mathrm{E}$ & 0 & $(1.8 \pm 0.2) \cdot 10^{2}$ \\
\hline Belgrade/Serbia [14] & $44.51 \mathrm{~N}$ & $20.23 \mathrm{E}$ & 0 & $(1.37 \pm 0.06) \cdot 10^{2}$ \\
\hline Slanic/Romania [5] & $45.24 \mathrm{~N}$ & $25.94 \mathrm{E}$ & 0 & $(1.43 \pm 4.9) \cdot 10^{2}$ \\
\hline Belgrade/Serbia & $44.51 \mathrm{~N}$ & $20.23 \mathrm{E}$ & 25 & 45 \\
\hline Belgrade/Serbia & $44.51 \mathrm{~N}$ & $20.23 \mathrm{E}$ & 75 & 11.9 \\
\hline Belgrade/Serbia & $44.51 \mathrm{~N}$ & $20.23 \mathrm{E}$ & 100 & 7.24 \\
\hline Ogoya/Japan [15] & $36.20 \mathrm{~N}$ & $136.5 \mathrm{E}$ & 270 & $5 \cdot 10^{-1}$ \\
\hline Belgrade/Serbia & $44.51 \mathrm{~N}$ & $20.23 \mathrm{E}$ & 300 & $7.9 \cdot 10^{-1}$ \\
\hline Slanic/Romania & $45.24 \mathrm{~N}$ & $25.94 \mathrm{E}$ & 601 & $(1.9 \pm 0.2) \cdot 10^{-1}$ \\
\hline Slanic/Romania & $45.24 \mathrm{~N}$ & $25.94 \mathrm{E}$ & 610 & $(1.8 \pm 0.1) \cdot 10^{-1}$ \\
\hline Slanic/Romania & $45.24 \mathrm{~N}$ & $25.94 \mathrm{E}$ & 790 & $(9 \pm 1.0) \cdot 10^{-2}$ \\
\hline CUPP/Finland & $63.39 \mathrm{~N}$ & $26.02 \mathrm{E}$ & 980 & $(2.1 \pm 0.2) \cdot 10^{-2}$ \\
\hline Solotwina/Ukraine [16] & $50.11 \mathrm{~N}$ & $23.16 \mathrm{E}$ & 1000 & $1.7 \cdot 10^{-2}$ \\
\hline LSC/Spain [17] & $42.43 \mathrm{~N}$ & $00.31 \mathrm{E}$ & 1200 & $4 \cdot 10^{-3}$ \\
\hline Oto-Cosmo/Japan [18] & $34.41 \mathrm{~N}$ & $135.5 \mathrm{E}$ & 1400 & $4 \cdot 10^{-3}$ \\
\hline Carlsbad/USA [19] & $33.09 \mathrm{~N}$ & $117.2 \mathrm{~W}$ & 1526 & $4.73 \cdot 10^{-3}$ \\
\hline CUPP/Finland & $63.39 \mathrm{~N}$ & $26.02 \mathrm{E}$ & 1900 & $(3.2 \pm 0.3) \cdot 10^{-3}$ \\
\hline Y2L/Korea [20] & $38.04 \mathrm{~N}$ & $128.4 \mathrm{E}$ & 2000 & $2.7 \cdot 10^{-3}$ \\
\hline Soudan/USA [21] & $47.49 \mathrm{~N}$ & $92.14 \mathrm{~W}$ & 2000 & $2 \cdot 10^{-3}$ \\
\hline LSC/Spain & $42.43 \mathrm{~N}$ & $00.31 \mathrm{E}$ & 2400 & $2 \cdot 10^{-3}$ \\
\hline Kamioka/Japan [22] & $39.29 \mathrm{~N}$ & $140.3 \mathrm{E}$ & 2700 & $3 \cdot 10^{-3}$ \\
\hline Boulby/UK [23] & $54.34 \mathrm{~N}$ & $00.58 \mathrm{~W}$ & 2800 & $4.05 \cdot 10^{-4}$ \\
\hline CUPP/Finland & $63.39 \mathrm{~N}$ & $26.02 \mathrm{E}$ & 2810 & $(6.2 \pm 0.6) \cdot 10^{-4}$ \\
\hline Gran Sasso/Italy [24] & $42.48 \mathrm{~N}$ & $13.33 \mathrm{E}$ & 3200 & $(3.41 \pm 0.01) \cdot 10^{-4}$ \\
\hline CUPP/Finland & $63.39 \mathrm{~N}$ & $26.02 \mathrm{E}$ & 3960 & $(1.1 \pm 0.1) \cdot 10^{-4}$ \\
\hline BNO/Russia [25] & $43.40 \mathrm{~N}$ & $43.32 \mathrm{E}$ & 4700 & $3 \cdot 10^{-5}$ \\
\hline Frejus/France [26] & $45.08 \mathrm{~N}$ & $06.41 \mathrm{E}$ & 4800 & $4.7 \cdot 10^{-5}$ \\
\hline Sudbury/USA [27] & $46.28 \mathrm{~N}$ & $81.10 \mathrm{~W}$ & 6010 & $3 \cdot 10^{-6}$ \\
\hline
\end{tabular}

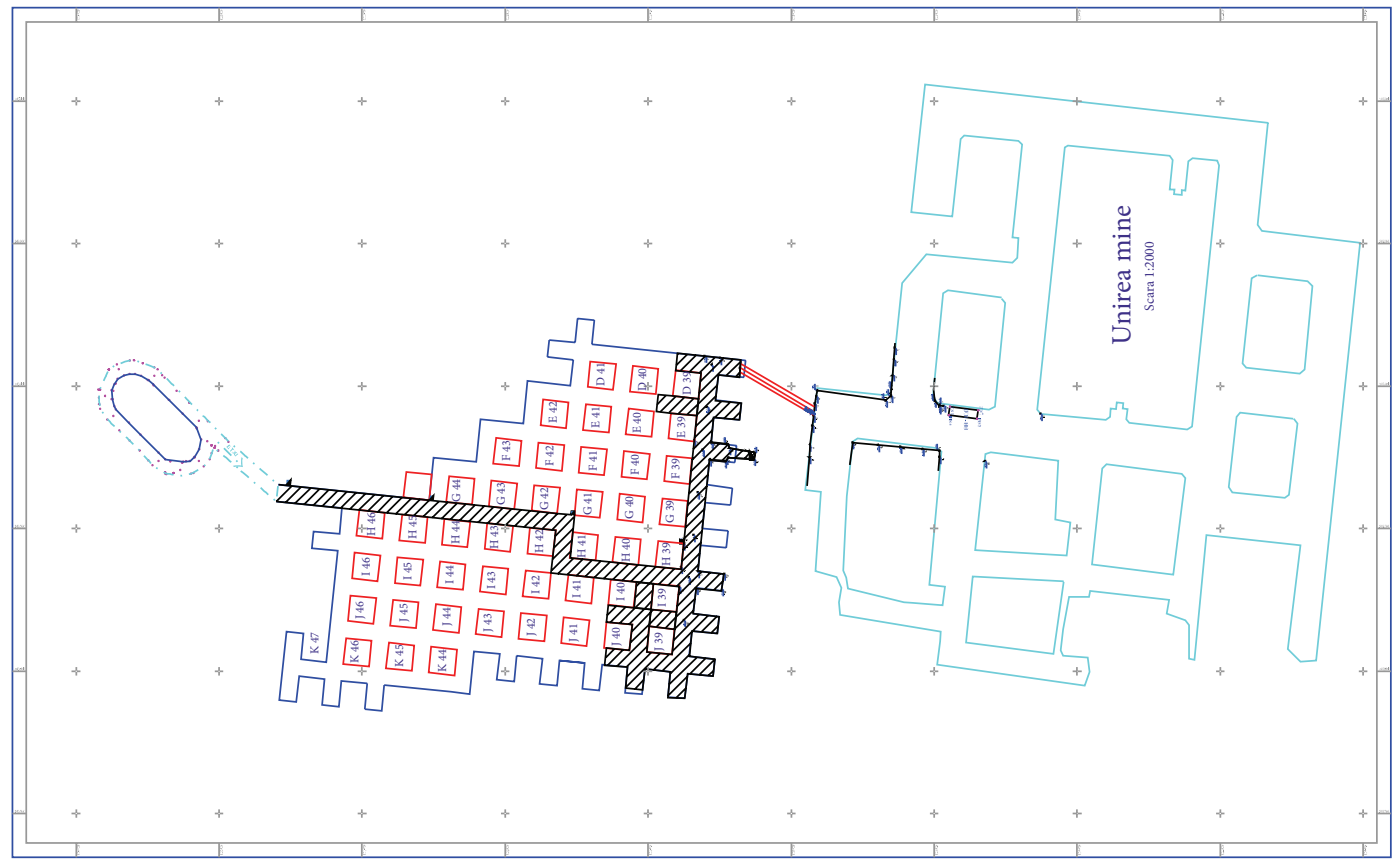

FIgURE 7: Map of Unirea mine, with the mBq laboratory of IFIN-HH. 
TABLE 3: Rate of coincidences between sheets. Measurements were performed in the Unirea mine $(-208 \mathrm{~m}$ depth from surface). The acquisition time for each measurement was set to 10 hours. The coincidence is represented by " $\cap$ " sign.

\begin{tabular}{lc}
\hline Channels & Counting rate $(\mathrm{p} / \mathrm{s})$ \\
\hline$(1+2+3+4) \cap(5+6+7+8)$ & $(191 \pm 2.3) \cdot 10^{-3}$ \\
$1+6$ & $(5 \pm 0.4) \cdot 10^{-3}$ \\
$4+7$ & $(8 \pm 0.5) \cdot 10^{-3}$ \\
$2+5$ & $(4 \pm 0.3) \cdot 10^{-3}$ \\
$3+8$ & $(6 \pm 0.4) \cdot 10^{-3}$ \\
$2+7$ & $(4 \pm 0.3) \cdot 10^{-3}$ \\
$3+6$ & $(5 \pm 0.4) \cdot 10^{-3}$ \\
$(1+2) \cap(7+8)$ & $(9 \pm 0.5) \cdot 10^{-3}$ \\
$(3+4) \cap(5+6)$ & $(26 \pm 0.8) \cdot 10^{-3}$ \\
\hline
\end{tabular}

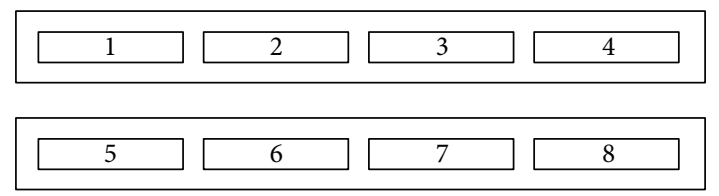

FIGURE 8: The orientation of the scintillator sheets of the new detector setup.

placed near the wall of the mine and sheets 4 and 8 toward the empty space of the cavern.

We can observe that the coincidence rate between $3+4$ and $5+6$ is 3 times higher than the coincidence rate of $1+2$ and $7+8$, indicating the fact that the muons which are arriving at the detector from the direction of the cavern are more numerous than those which are coming from the direction of the wall. This is an important observation because in this way we can determine the variations of the density in different materials or in the terrestrial crust, with many practical applications, like the detection of oil deposits, hidden rooms in pyramids, or at customs checkpoints in the search of contraband products.

\section{Conclusions}

A versatile detector for cosmic muon flux measurements was set up and studied at IFIN-HH, Bucharest. It can be used in many applications where the information about the flux of atmospheric muons is of interest. One of them that were demonstrated in this paper is the determination of water equivalent depths in Unirea mine of Slanic [9] and could be used to measure the water equivalent depth on any site in order to check the possibility to install a large underground experiment like LAGUNA, MINOS [12], and so forth. The results show that the mobile detector could be used to measure the directional variation of the muon flux in order to study hadronic interaction models or muon multiplicities.

In this work, the technique of collecting light signals by PMTs through optical fibers was investigated. Its reliability was demonstrated by the good agreement between the results obtained with this configuration and another one, where the light is transferred from the scintillator plate to a PMT through a light guide, in the determination of the cosmic muon flux in underground locations (Unirea mine from Slanic Prahova) and the variation of muon flux with the altitude. This type of reading technique combined with the mobility of the detector could be used easily in various applications, like archaeology and volcanology, or as a scanner used in customs control.

\section{Acknowledgment}

The Romanian authors would like to thank the support of the Romanian Authority for Scientific Research by the Projects Parteneriate 194/2012_CORONA and PN 09370105.

\section{References}

[1] A. Rubbia, "The LAGUNA Design Study: towards giant liquid based underground detectors for neutrino physics and astrophysics and proton decay searches," Acta Physica Polonica B, vol. 41, pp. 1727-1732, 2010.

[2] A. Saftoiu, A. Bercuci, I. M. Brancus et al., "Measurements of the cosmic muon flux with the willi detector as a source of information about solar events," Romanian Journal of Physics, vol. 56, no. 5-6, pp. 664-672, 2011.

[3] J. Marteau, D. Gibert, N. Lesparre, F. Nicollin, P. Noli, and F. Giacoppo, "Muons tomography applied to geosciences and volcanology," Nuclear Instruments and Methods in Physics Research, vol. 695, no. 11, pp. 23-28, 2012.

[4] S. Aguillar et al., "Searching for cavities in the Teotihuacan Pyramid of the Sun using cosmic muons," in Proceedings of the 32nd International Cosmic Ray Conference (ICRC '11), Beijing, China, 2011.

[5] B. Mitrica, R. Margineanu, S. Stoica et al., "A mobile detector for measurements of the atmospheric muon flux in underground sites," Nuclear Instruments and Methods in Physics Research A, vol. 654, pp. 176-183, 2011.

[6] H. Bozdog, M. Elzerb, H.J. Gils et al., “The detector system for measurement of multiple cosmic muons in the central detector of KASCADE," Nuclear Instruments and Methods in Physics Research A, vol. 465, pp. 455-471, 2001.

[7] T. Antoni et al., "The cosmic-ray experiment KASCADE," Nuclear Instruments and Methods in Physics Research A, vol. 513, pp. 490-510, 2003.

[8] D. Heck, J. Knapp, J. N. Capdevielle, G. Schatz, and T. Thouw, "CORSIKA: a Monte Carlo code to simulate extensive air showers," Report FZKA 6019, Forschungszentrum, Karlsruhe, Germany, 1998.

[9] J. A. Formaggio and C. J. Martoff, "Backgrounds to sensitive experiments underground," Annual Review of Nuclear and Particle Science, vol. 54, pp. 361-412, 2004.

[10] V. A. Kudryavtsev, "Muon simulation codes MUSIC and MUSUN for underground physics," Computer Physics Communications, vol. 180, no. 3, pp. 339-346, 2009.

[11] R. Margineanu, C. Simion, S. Bercea et al., "The Slanic-Prahova (ROMANIA) underground low-background radiation laboratory," Applied Radiation and Isotopes, vol. 66, no. 10, pp. 15011506, 2008.

[12] P. Schreiner et al., "Interpretation of the atmospheric muon charge ratio in MINOS," in Proceedings of the30th International 
Cosmic Ray Conference (ICRC '07), vol. 9, pp. 97-98, Merida, Mexico, 2007.

[13] T. Enqvist, A. Mattila, V. Föhr et al., "Measurements of muon flux in the Pyhäsalmi underground laboratory," Nuclear Instruments and Methods in Physics Research A, vol. 554, pp. 286-290, 2005.

[14] A. Dragic, D. Jokovića, R. Banjanaca et al., "Measurement of cosmic ray muon flux in the Belgrade ground level and underground laboratories," Nuclear Instruments and Methods in Physics Research A, vol. 591, pp. 470-475, 2008.

[15] K. Komura and Y. Hamajima, "Ogoya underground laboratory for the measurement of extremely low levels of environmental radioactivity: review of recent projects carried out at OUL," Applied Radiation and Isotopes, vol. 61, pp. 185-189, 2004.

[16] Y. G. Zdesenko et al., "The study of the background of the detectors in the Solotvina underground laboratory," in Proceedings of the 2nd International Symposium on Underground Physics, vol. 1987, p. 291, Baksan Valley, Russia, 1988.

[17] A. Bettini, "The Canfranc Underground Laboratory (LSC)," The European Physical Journal Plus, vol. 127, article 112, 2012.

[18] H. Ohsumi, H. Ejiri, M. Fujiwara et al., "The new underground laboratory, "Oto Cosmo Observatory" and ELEGANT Detectors," in Proceedings of the XV RCNP Osaka International Symposium on Nuclear Physics Frontiers with Electro-Weak Probes, pp. 274-279, World Scientific, March 1996.

[19] E.-I. Esch, T.J. Bowles, A. Hime, A. Pichlmaier, R. Reifarth, and H. Wollnik, "The cosmic ray muon flux at WIPP," Nuclear Instruments and Methods in Physics Research A, vol. 538, pp. 516-525, 2005.

[20] J. J. Zhu, K. J. Kang, Y. J. Li et al., "Study on the muon background in the underground laboratory of KIMS," High Energy Physics and Nuclear Physics, vol. 29, pp. 721-726, 2005.

[21] W. W. M. Allison et al., "Cosmic ray sun shadow in soudan 2 underground muon flux," in Proceedings of the 26th International Cosmic Ray Conference, August 1999.

[22] Y. Fukuda, T. Hayakawa, E. Ichihara et al., "Measurement of the flux and zenith-angle distribution of upward throughgoing muons by super-kamiokande," Physical Review Letters, vol. 82, pp. 2644-2648, 1999.

[23] M. Robinson, V. A. Kudryavtsev, R. Lüscher et al., "Measurements of muon flux at $1070 \mathrm{~m}$ vertical depth in the Boulby underground laboratory," Nuclear Instruments and Methods in Physics Research A, vol. 511, pp. 347-353, 2003.

[24] G. Bellini, G. J. Benzigerf, D. Bick et al., "Cosmic-muon flux and annual modulation in Borexino at $3800 \mathrm{~m}$ water-equivalent depth," Journal of Cosmology and Astroparticle Physics, vol. 2012, no. 15, article 5, 2012.

[25] Y. M. Andreyev, V. I. Gurentsov, and I. M. Kogai, "Seasonal and diurnal variations of cosmic ray intensity based on measurements at depth of approximately $8.5 * 10^{4} \mathrm{~g}^{2} \mathrm{~cm}^{-2}$, in Proceedings of the 20th International Cosmic Ray Conference (ICRC '87), vol. 6, p. 200, Moscow, Russia, 1987.

[26] W. Rhode, "Measurements of the muon-flux with the Fréjusdetector," Nuclear Physics B, vol. 35, pp. 250-253, 1994.

[27] B. Aharmim, S. Ahmed, T. Andersen et al., "Measurement of the cosmic ray and neutrino-induced muon flux at the Sudbury neutrino observatory," Physical Review D, vol. 80, Article ID 012001, 15 pages, 2009.

[28] R. Brun and F. Carminati, GEANT Detector Description and Simulation Tool, CERN Programming Library Long Writeup W5013, 1993.
[29] S. Agostinelliae, J. Allison, and K. Amako, "Geant4-a simulation toolkit," Nuclear Instruments and Methods in Physics Research A, vol. 506, pp. 250-303, 2003.

[30] K. Greisen et al., "The intensities of the hard and soft components of cosmic rays as functions of altitude and zenith angle," Physical Review, vol. 61, pp. 212-221, 1942. 

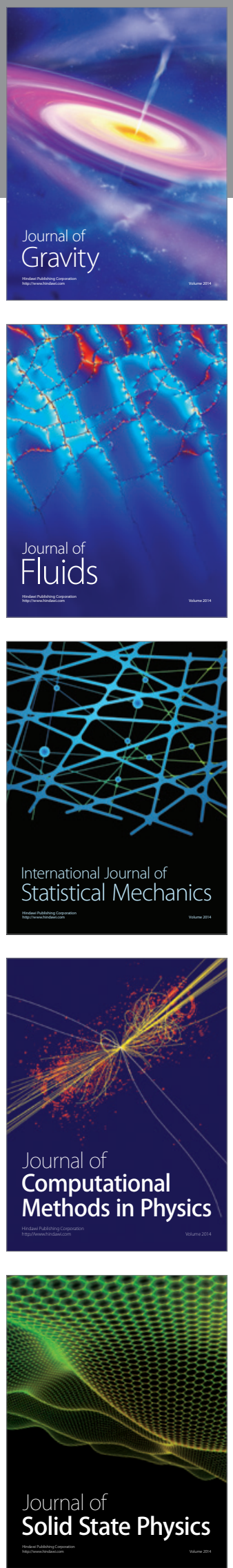

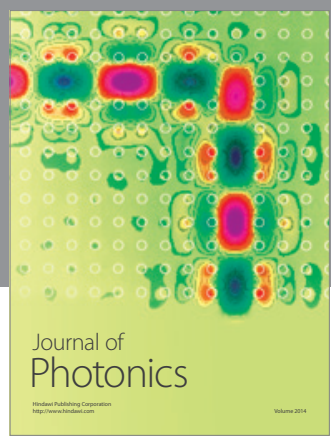

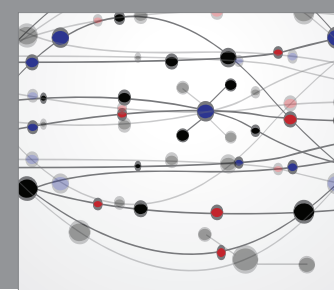

The Scientific World Journal

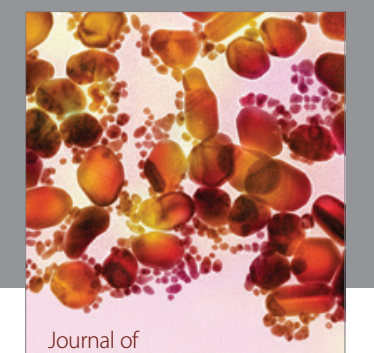

Soft Matter
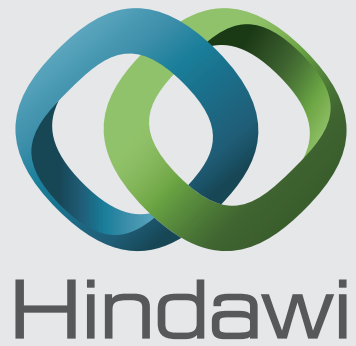

Submit your manuscripts at

http://www.hindawi.com
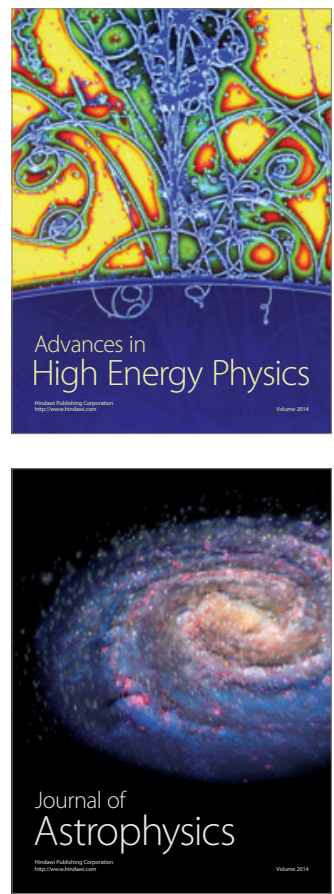
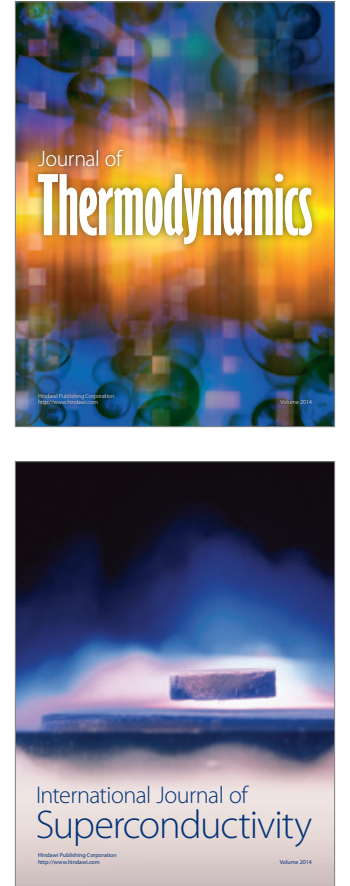
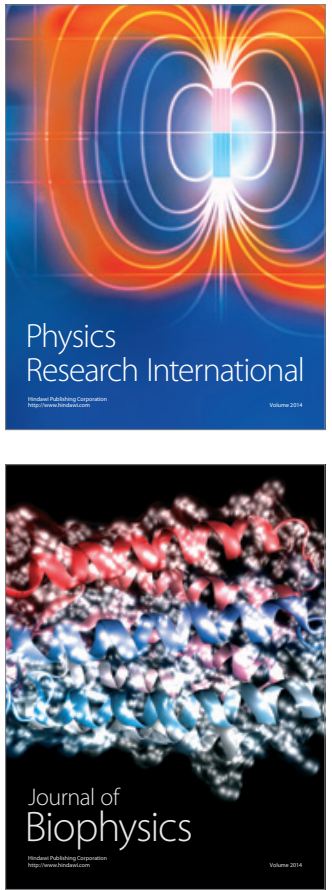
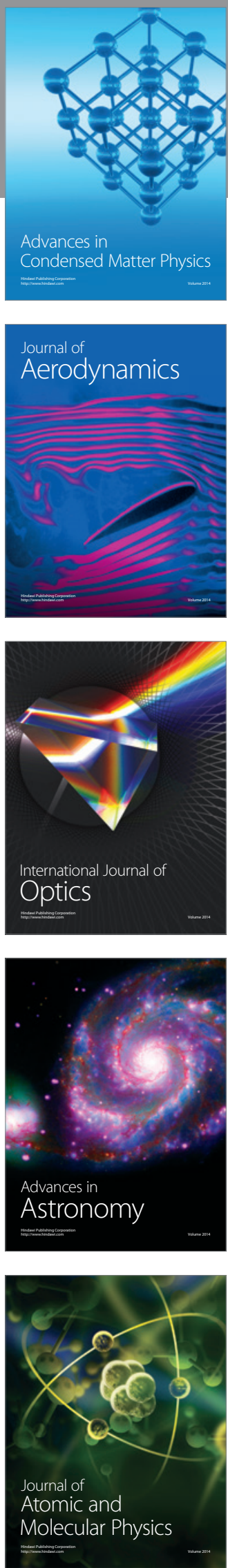